\title{
A DETERMINISTIC AND RANDOM ERROR MODEL FOR A MULTIBEAM HYDROGRAPHIC SONAR SYSTEM
}

Richard D. Angelari, PhD

\author{
National Oceanic and Atmospheric Administration \\ National Ocean Survey C651 \\ Test and Evaluation Laboratory \\ Sensor Test Branch \\ Rockville, Maryland 20852
}

Abstract

The expanding and increasing affluent world population has brought about a growing need for commodities and energy on a global scale. Two major marine activities affected by this are shipping and mining. The shipping industry, because of its up to 30 meter draft superships, and the mining industry (particularly the hydrocarbon sector), both require timely and accurate bathymetric charts for safe and economical operation. To meet these needs the Bathymetric Swath Survey System $\left(\mathrm{BS}^{3}\right)$ is being developed. The BS3 is a 21 beam mapping sonar designed to accurately and efficiently portray the seabed directly beneath and to the port and starboard side of an underway hydrographic ship. This paper examines the effects of four major inherent error sources on system accuracy. These sources are uncompensated ship rol1, sound velocity errors, pulse stretching and noise.

\section{Introduction}

The $\mathrm{BS}^{3}$ is a 21 -beam sonar system designed to measure a swath of ocean bathymetry during hydrographic missions. Figure 1 depicts the swath geometry. Basically, the system operates in two modes, the transmit mode and the receive mode. During one transmit mode, the portside bottom is insonified by an acoustic pulse, followed by a portside receive mode. The receive mode utilizes 11 contiguously directed $5^{\circ}$ beams, one vertical beam, and 10 portside beams. The portside operation therefore consists of a total swath coverage of $55^{\circ}$. These operating modes are then repeated for the starboard side. Depth sensed by each beam is determined by measuring the go-return time of the acoustic pulses and transforming this time interval to slant ranges to the bottom. These slant ranges in turn are then translated to depths by a simple trigonometric operation. Errors due to ship's motion and environmental factors are for the most part sensed by the BS 3 signal processing subsystems and corrected for, however, limitations of the sensing elements, electronics, and noise may contribute errors. The establishment of the error bounds due to these sources is the objective of this study.

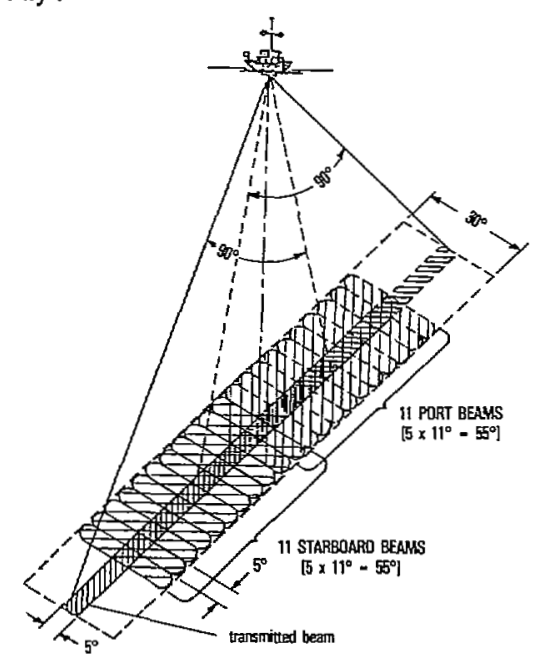

Figure 1. Bathymetric Swath Survey System (BS3)

\section{Error Sources}

Error sources will be designated as follows:

Type I error (uncompensated roll error) is that portion of ship roll not sensed by the ship roll sensor (due to its inherent limitations) and therefore uncompensated by the $\mathrm{BS}^{3}$ processing system. This error will be symbolized by $\Delta \theta_{p}$.

Type II error (sound speed error) is error resulting from less than perfect estimates of sound speed in water. This error is expressed as a ratio of tine measured velocity to true velocity $C^{*}=\frac{C \text { measured }}{C \text { true }}$.

Type III error (pulse stretching error)

It will be demonstrated in the discussion to follow, although the return echo is time delayed and attenuated, it is not a replica of the idealized pulse, i.e., having infinite slope at its leading and trailing edges. It is, in fact, attenuated and in addition, it is rounded and stretched. This distortion results in delaying the indicated time of arrival of the return echo and is therefore an error source. 
Type IV error (noise related error) error is similar in effect to pulse stretching error since it causes premature indication (instead of delayed as for pulse stretching) of the pulse arrival time and is therefore considered an error of the same rank. Figure $2 a$ shows a ship's hull together with the necessary geometry to establish the error model. Figure $2 b$ depicts the pulse stretching effect. The inner beam (vertical beam) receives a relatively narrow pulse with relatively straight edges. The outer beam return pulse is much more rounded and stretched. The slope of the leading edge decreases as the pulse width increases. This becomes apparent by the following argument. Consider a beam pointing at an angle $\theta p$ degrees from the vertical as indicated. $\theta_{\mathrm{BW}}$ is the beam width angle. The center of the beam strikes the bottom at $D$, the inner edge at $A$, the outer edge at $C$. AB represents a wave front. The inner edge arrives at the bottom first whereas the outer edge does so at some time later equal to the time of propagation along $X^{*}$. Thus points $A \xi B$ on the $E \%$ incoming wave front which traveled together at launch time arrive back at the receiver separated from each other by the propagation time to traverse distance $2 X$. The pulse has therefore been stretched. This stretching decreases the edge slopes of the return pulse.

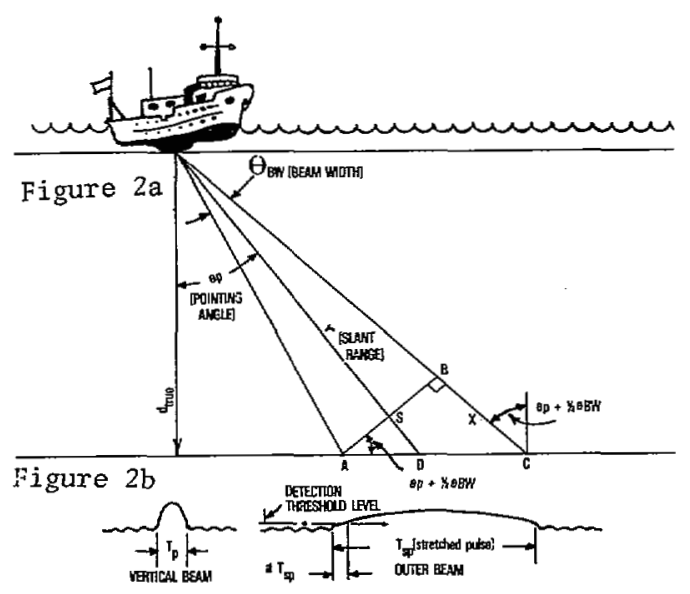

Since the detection threshold level is fixed, pulse detection time will vary as the pulse stretches. As a first approximation, it will be assumed that the error is proportional to the stretched pulse $\left(\mathrm{T}_{\mathrm{sp}}\right)$, i.e., detection time error is $\mathrm{T}_{\mathrm{sp}}$ where $a$ is a positive fractional number less than 1 and is hereon designated as the timing error coefficient. can also take on negative values; this follows since the stretched pulse is in a noisy environment and premature triggering can occur if noise peaks exceed the threshold level for any appreciable time prior to the true arrival time of the return echo.

\#X represents the distance $B C$.

\section{Mathematical Error Mode1}

Type I, II, III, IV Error Model - Depth error can be expressed as a \% quantity as follows:

$$
\varepsilon \%=\frac{\text { True Depth- Measured Depth }}{\text { True Depth }} \times 100 \%
$$

where $E \%$ is the error in $\%$ relative to the true depth.

To establish the contribution to depth error due to error sources, a functional relationship between these sources and depth error in $\%$ is desired, that is:

$$
e \%=F\left\{\Delta \theta_{p}, C^{*}, \frac{d}{d}\right\}
$$

This relationship is: (Angelari [1])
Equation (3) is the mathematical error model for the $\mathrm{BS}^{3}$ system having Type I, II, III, and IV. error sources.

\section{Error Analysis}

Deterministic vérsus Random Error Sources:

Central to the analysis are the concepts of deterministic phenomena and random phenomena. Deterministic phenomena are those that can be described by an explicit mathematical relationship. Random phenomena are those that can be described only in terms of probability statements and statistical averages.

The error mode1, Equation (3), may represent either deterministic or random system error depending on the nature of the error sources. If the error sources $\Delta \theta_{\mathrm{p}} \mathrm{C}^{*}$, and $\mathrm{f}^{\mathrm{f}}$ are deterministic then this equation is the $B^{3}$ Deterministic Error Model (DEM). If the sources are random then this equation represents the Random Error Model (REM). Both types may exist simultaneously, for this reason both will be considered in this study.

Estimates of the expected ranges of error sources are:

$$
\begin{aligned}
\Delta \theta_{\mathrm{p}} & = \pm 1^{\circ} \text { to } \pm 3^{\circ} \\
d & = \pm 0.1 \text { to } \pm 0.3 \\
\mathrm{C}^{*} & =1 \pm .01 \text { to } 1 \pm .02
\end{aligned}
$$

These will be denoted as the specified error sources (SES) and will be designated as follows:

$$
\Delta \theta p=+1^{\circ}, \not=+0.1 \text { and } \mathrm{C}^{*}=\underline{1 \pm .01} \text { "1o error" SES }
$$


$\theta p= \pm 3^{\circ}, A=+0.3$ and $C^{*}= \pm 1.02$ "hi error" SES

The SES will first be considered as deterministic errors and will be used in the DEM analysis to follow. For the REM analysis "lo error" (i,e., $\Delta \theta p=+1^{\circ}, \not a=+0.1$ and $\left.C^{*}=1.01\right)$ will be specified as the statistical descriptors and represent the $1 \sigma$ values for the uncompensated roll, sound velocity, pulse stretching and noise error sources.

\section{Deterministic Error ModeI}

Plots of the error model (eq 3 ) for ranges of error source magnitudes will be informative. To decrease the numbex of plots the outer beam $\theta_{\psi}^{*}=50^{\circ}$ and the inner beam $\theta_{\psi}=0^{\circ}$ only will be plotted and only the maximum roll specified for the BS3 $\left(20^{\circ}\right)$ will be treated. Thus a total of four pointing angles will be considered. These are shown in Table $I$.

Table I

POINTING ANGLES EVALUATED IN THIS STUDY

$$
\begin{aligned}
& \text { 1. } \theta \mathrm{p}=0^{\circ} \text { vertical beam } \\
& \text { 2. } \theta \mathrm{p}=20^{\circ} \text { (vertical beam }+20^{\circ} \text { rol1) } \\
& \text { 3. } \theta \mathrm{p}=50^{\circ} \text { outer beam } \\
& \text { 4. } \theta \mathrm{p}=70^{\circ} \text { (outer beam }+20^{\circ} \text { roll) }
\end{aligned}
$$

Plots of the $\mathrm{BS}^{3}$ system model error due to the SES are shown in Figures 3 through 8 , $\varepsilon_{\%}$ is plotted along the ordinate, $\Delta \theta p$ along the abscissa, $\not \nexists$ as a parameter and for fixed values of $\mathrm{C}^{*}$. The rhomboids in these figures delimit the error bounds for "lo error" (mnop) and "hi error" (rstu) sources.

\section{Discussion of Error Curves}

Case 1 . The $50^{\circ}$ bean with $+20^{\circ}$ rol1, $\left(\theta \mathrm{p}=70^{\circ}\right)$ $\mathrm{C}^{*}=1.00$ (Figure 3 )

Figure 3 is the error diagram for the outermost beam $\left(\theta_{\Psi}=50^{\circ}\right)$ with a positive $20^{\circ}$ roll, i.e., $\theta p=70^{\circ}$. In addition, it is assumed here that the sound speed has been correctly estimated, i.e. $C^{*}=1.00$. The rhomboid mnop represents the operating error region for "lo error", i.e., for $\Delta \theta \mathrm{p}= \pm 1^{\circ}$ and $\neq+0.1$. From this diagram it is concluded that the measured depth error will not be greater than between $6.7 \%$ positive (point $n$ ) or $7.8 \%$ negative (point $p$ ) for " 10 " excursions. Thus a true depth of 100 feet would be indicated as being anywhere between 93.3 feet and 107.8 feet. For "hi error," $\theta p= \pm 3^{\circ}, \quad \alpha= \pm 0.3$, the range of error is between $17.9 \%$ (s) and approximately $-30 \%(u)$. For this case the true depth would be indicated as being anywhere between 82.1 feet to 130 feet. Clearly the more serious errors are the ones having the greatest negative magnitudes since hazards at the true depth could be indicated to be deeper than they are, i.e., 100 feet true depth may be indicated to be 130 feet deep. The diagram also shows that for $50 \%$ of the pointis the fixed array beam ang $1 e, \theta p$ is the
poing time the excursion is in the negative regions [3rd and 4th quadrants) meaning that the more serious type error is as probable as the less serious (positive regions) error. Point $q$ is the quiescent operating point and represents a bias due to error in estimating sound velocity with no other sources present. In this case, since the estimate of sound speed was correct, the static system bias error is zero.*

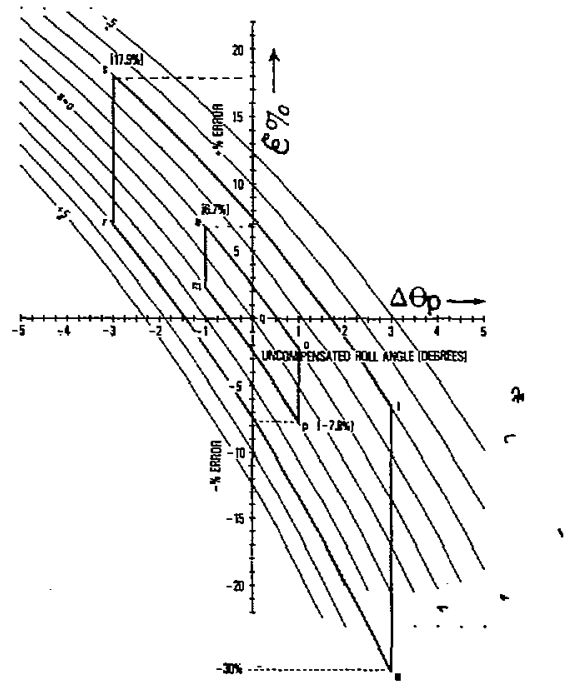

Figure $3 \quad \theta p=70^{\circ}, C^{*}=1.00$

Case 2. The Vertical Beam with $0^{\circ}$ Roll, $\theta p=0$, $\overline{\mathrm{C}^{*}=1}$ (Figure 4)

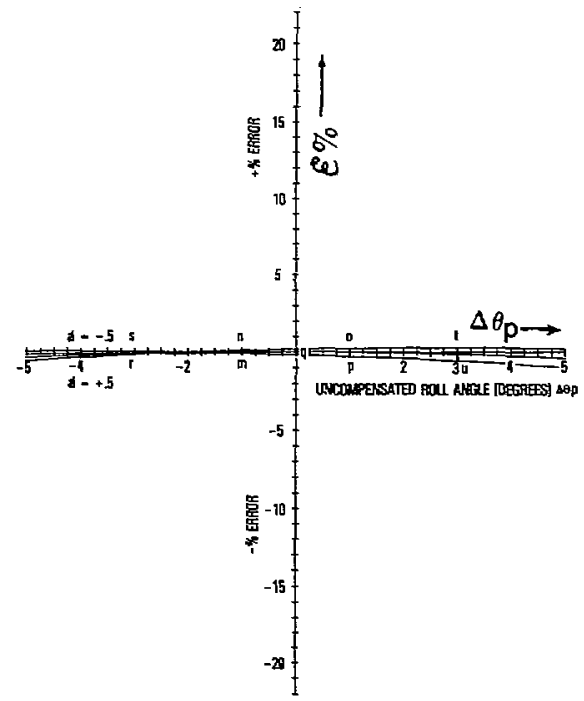

Figure $4 \theta p=0, C^{*}=1.00$

* It is not meant here that the $\mathrm{BS}^{3}$ error is zero when $\mathrm{C}^{*}=1$ but rather the MODEL bias error is zero. It is expected that an overall system bias error exists and will show itself when the BS 3 field tests are completed. 
The rhomboid area has diminished greatly indicating a gxeatly reduced error mode of operation. This is to be expected since the return echo in the vertical beam experiences the least pulse stretching (see Figure $2 \mathrm{~b}$ ). Note that for this case the system error is almost independent of the magnitude of $a$. The maximum error, (for "hi error") is less than $+0.5 \%$. The rhomboid degenerates to a point (the $q$ point) for $d=0$ and $\Delta \theta p=0$ and since $C^{*}=1$ (for this case) $\varepsilon_{\%}^{\circ}$ is zero, i.e., an errorless system.

Case 3 . The vertical beam with $20^{\circ}$ roll i.e., $\theta p=$ $0^{\circ} \mathrm{C}^{*}=1.00$ (Figure 5).

The rhomboid area has increased such that "lo error" and "hi error" are approximately $\pm 1^{\circ}$ and $\pm 2 \%$ respectively.

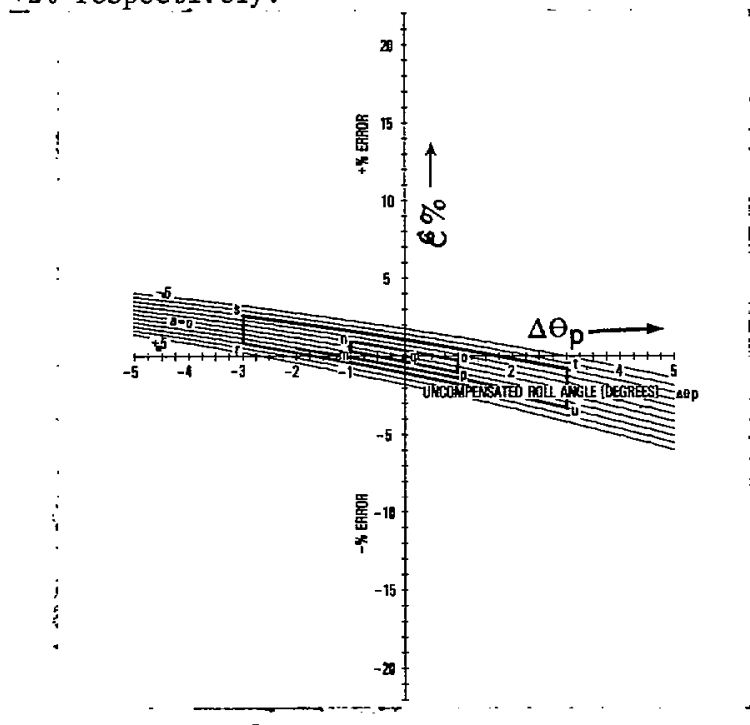

Figure $5 \theta \mathrm{p}=20^{\circ}, \mathrm{C}^{*}=1.00$

Case 4. The $50^{\circ}$ beam with $0^{\circ}$ roll i.e., $\theta p=50^{\circ}$; $\overline{\mathrm{C}^{*}=1} . \overline{0} 2$ (Figure 6).

This figure depicts system error with $0^{\circ}$ roll and an overestimation of sound speed of $+2 \%$. The quiescent point has shifted negatively by $2 \%$ this is a systematic error. The "lo" rhomboid is now almost entirely in the negative quadrants, this is the most undesirable error since this would indicate a potential hazard to be deeper than its true depth.

Case 4. The vertical beam with $0^{\circ}$ roll $C^{*}=1.02$ (Figure 7).

The rhomboid are significantly decreased, the quiescent point is again at $-2 \%$. Uncompensated rolls of magnitude up to $3^{\circ}$ contribute little error, here again this is due to the near negligible effect on the vertical beam of pulse strefching as previously discussed (Fig. 2b). Figure $7 b$ shows the biasing effect in the vertical due to error in estimating sound speed by $+5 \%\left(C^{*}=1+.05\right)$. The case for $0 \%$ error $\left(C^{*}=1.00\right)$

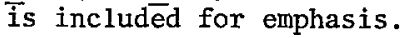

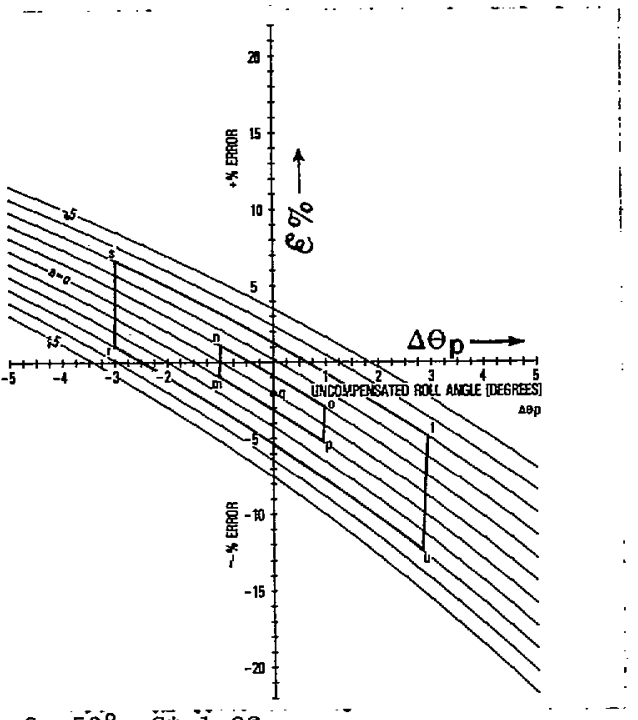

Figure ${ }^{-} 6 \quad \theta p=50^{\circ}, \overline{\mathrm{C}}^{*}=\overline{\mathrm{i}} .02$

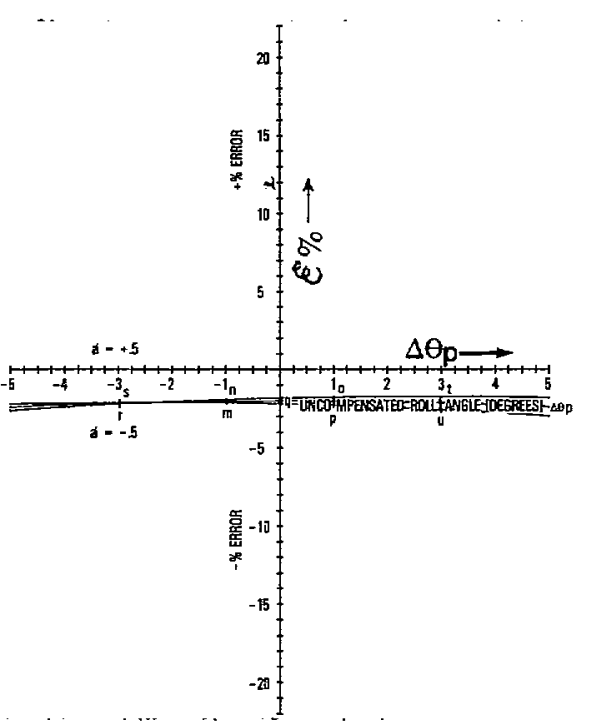

Figure $7 a \quad \theta p=0^{\circ}, c^{\star}=1.02$

A passing note is made to indicate the role played by the beam-width arigle ${ }{ }_{B W}$ As can be verifjed by reference to equation (3), a reduction of beamwidth from the present $5^{\circ}$ to $1^{\circ}$ will reduce sensitivity to (pulse stretching and noise effect). This in effect would have a marked improvement on system performance. Since decreased beam-width requires larger transducer elements, among other factors, a trade-off study would be necessary should such an improvement be sought. The highlighted rhomboid (Figure 8) represents "lo error" regicn for the outer bean with $20^{\circ}$ roll $\left(\theta p=70^{\circ}\right.$ ) $\mathrm{C}^{*}=100$ and $\theta_{\mathrm{BW}}=1^{\circ}$. This figure represents the mitude error sources of figure 3 but with a 5 to 1 reduction in beam-width $\left(i, e\right.$. from $5^{\circ}$ to $\left.1^{\circ}\right)$. 


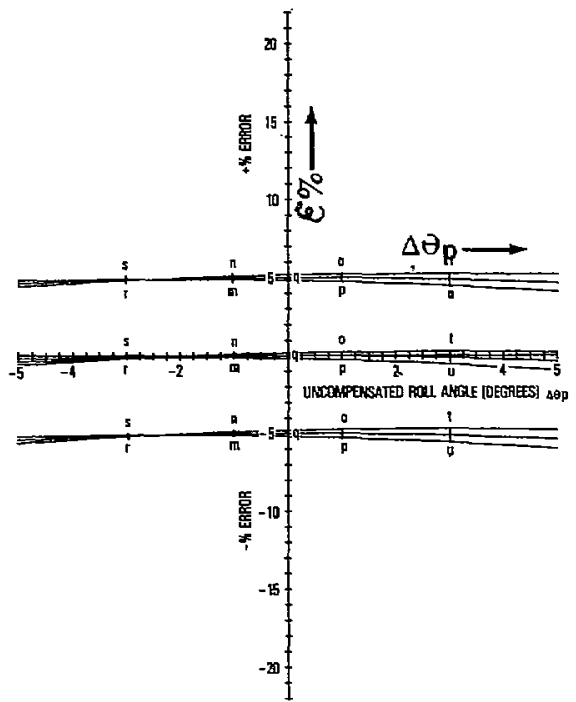

Figure $760 p=0^{\circ}, C^{*}-1.00,1.05$ and 0.95

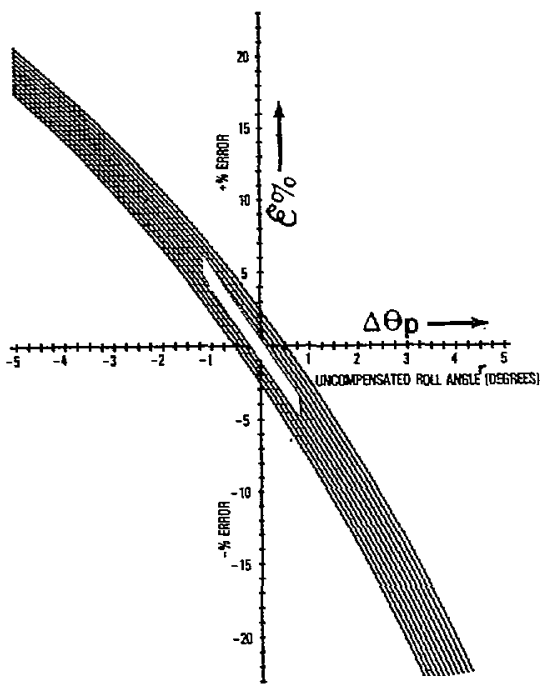

Figure 8 Effect of decreasing beamwidti from $5^{\circ}$ to $1^{\circ}$ for the outermost beam with $a p=79^{\circ}$, $\mathrm{C}^{*}=1.00$ (compare with Figure 3 )

Figure 9 is a graphical display (in bar-line graph form) summarizing the salient information of this study. This figure displays the following information for $\theta \mathrm{p}=70^{\circ}, 50^{\circ}, 20^{\circ}$, and $0^{\circ}$ for $\mathrm{C}^{*}=1.02,1.01,1.0,0.99$, and 0.98 .

1. The location of the operating point $q($ dot $)$

2. The error in \% of true depth due to a variation in and $\theta p$, for "lo exror" (bar ends)

3. The error in $\%$ of true depth to a variation in $a$ and $\theta p$, for "hi error" (1ine ends)

4. The operating area in the upper (clear areas) and lower (shaded areas) regions.

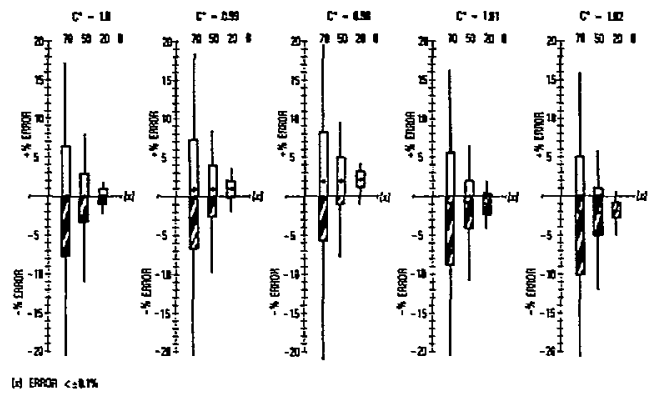

Figure 9 Bar-1ine graph summarizing "1o", "hi" error ranges. The dot represents the quiescent operating point ( $q$ point)

\section{Random Error Model}

For this model the error sources $\Delta p p$, $A$ and $C^{*}$ will be considered random variables normally distributed with zero mean and standard deviations equal to the SES "Io" error values. Formally stated this becomes:

$$
\begin{aligned}
& \begin{aligned}
\Delta \widetilde{P_{p}} & =N\left[0, \sigma_{\Delta \theta}^{2} \theta=N\left[0,\left(1^{0}\right)^{2}\right]\right. \\
\widetilde{A} & =N\left[0, \sigma^{2} \phi\right]=N\left[0,(0.1)^{2}\right]
\end{aligned} \\
& \widetilde{\mathrm{C}^{*}}=\mathrm{N}\left[0, \rho^{2}\right]=\mathrm{N}[0, \text { (1) } 2]
\end{aligned}
$$

(where a tilda $(\sim)$ indicates a random variable) Equation 3 may now be replaced by the corresponding random error model.

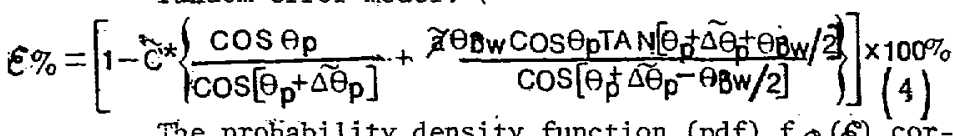

The probability density function (pdf) $f_{\theta}(\dot{\epsilon})$ cor responding to 4 , once found will completêly describe tise REP: Unfortunately, the density-

function may be very difficult or even impossitile to determine directly from Equation 4 . Equation 4 will be expanded about the zero point as a truncated Taylor series, a more tractable expression results, for small error excursions this becomes

$$
\varepsilon\left(\Delta \ddot{\theta} p, \ddot{n}, c^{*} d=p^{2}=p^{2} \dot{p}+q^{2}+r^{*}\right.
$$

where now $\theta p$, $\not$ and $C^{*}$ are realizations of the rvs and represent small excursions and

$$
p=\frac{\partial \stackrel{\varrho}{e}}{\partial \Delta \theta p} \quad q=\frac{\partial \varepsilon}{\partial \not} \quad r=\frac{\partial \varepsilon}{\partial c^{\star}}
$$

are partial derivatives and for saall excursions

a the tilda $(\sim)$ will be onitted it is understood we are dealing with random variables (rvs) 
are essentially constant. These partials are derived directly from Equation 4. A tabulation of $p, q$, and $r$ for each of the 11 pointing angles is given in Table II.

Table II

$\begin{array}{lccc}\theta \mathrm{p}^{\circ} & \mathrm{p} & \mathrm{q} & \mathbf{r} \\ 0 & 0 & -0.38 & -1.00 \\ 5 & -0.15 & -1.15 & -1.00 \\ 10 & -0.31 & -1.92 & -1.00 \\ 15 & -0.47 & -2.72 & -1.00 \\ 20 & -0.64 & -3.56 & -1.00 \\ 25 & -0.81 & -4.46 & -1.00 \\ 30 & -1.01 & -5.43 & -1.00 \\ 35 & -1.22 & -6.50 & -1.00 \\ 40 & -1.46 & -7.72 & -1.00 \\ 45 & -1.75 & -9.13 & -1.00 \\ 50 & -2.08 & -10.82 & -1.00\end{array}$

Since a linear combination of Gaussian terms is itself Gaussian,

$$
\varepsilon(\%)=N\left[{ }^{\mu} \varepsilon(\%), \sigma^{2} \varepsilon(\%)\right]
$$

where $\mu_{\varepsilon}(\%)=0$

$$
{ }^{2} \varepsilon(\%)=p^{2} \sigma_{\Delta y} p^{2}+q^{2} \sigma_{\alpha^{2}}+r^{2} \alpha^{2} c^{(7 b)}
$$

The two descrirtive parameters ${ }^{\mu} \varepsilon(\%)$ and $\sigma^{2} \hat{e}(\%)$ completely define the REH. Thus, probability of the $\mathrm{BS}^{3}$ error being between $+\delta$ is:

$$
P[-\delta(\%) \leq \varepsilon(\%) \leq \delta(\%)]=\frac{b}{2 \pi \sigma \varepsilon \mid(\%)} \int_{-\delta(\%)}^{+\delta(\%)} \exp \left[-\frac{\varepsilon^{2}(\%)}{2 \sigma^{2} \varepsilon(\%)}\right] d \varepsilon(\%)
$$

Integral (8) is a well tabulated function and may readily be evaluated, However, a more convenient method is to evaluate it graphically using normal probability paper. As an example consider the error predicted by the REM when the outermost beam angle is pointing at $50^{\circ}$, i.e. $\theta p=50^{\circ}$. From Table II :

$$
\begin{aligned}
& p=-2.08 \\
& q=-10.82 \\
& r=-1.00
\end{aligned}
$$

Substituting into Equation $7 \mathrm{~b}$

$$
\sigma_{\varepsilon}^{2}(\%)
$$

$\sigma_{\varepsilon}^{2}(\%)=(-2.08)^{2}\left[1^{\circ}\right]^{2}+(-10.82)^{2}[0.1]^{2}+(-1)^{2}[1]^{2}$

(the quantitics in the square [ ] brackets are the" 10 "SES)

from which

$$
\begin{aligned}
& \sigma_{\varepsilon}^{2}(\%)=6.59 \\
& \sigma_{\varepsilon}(\%)=2.75 \\
& \mu_{\varepsilon}(\%)=0
\end{aligned}
$$$$
\text { or } \quad \sigma_{\varepsilon}(\%)=2.75
$$$$
\text { I }
$$

also

II

Figure 10 is a plot of the REM (equation 4 ), it is seen that the $50^{\circ}$ beam when painting at $50^{\circ}$ may be in error by up to $+5.2 \%$ of depth at $95 \%$ CL.. Similarly, the vertical bean, $\theta p=0^{\circ}$, may be in error by $+1.9 \%$ of the true depth at the $95 \%$ CL. $\theta p=10^{\circ}, 20^{\circ}$, and $30^{\circ}$ plots are also shown. Intermediate pointing angle error plots may be established by calculating $\varepsilon \%$ using Equation $7 b$ and values of $p, q$, and $r$ for the desired $\theta p$. The plots also indicate the lo error values.

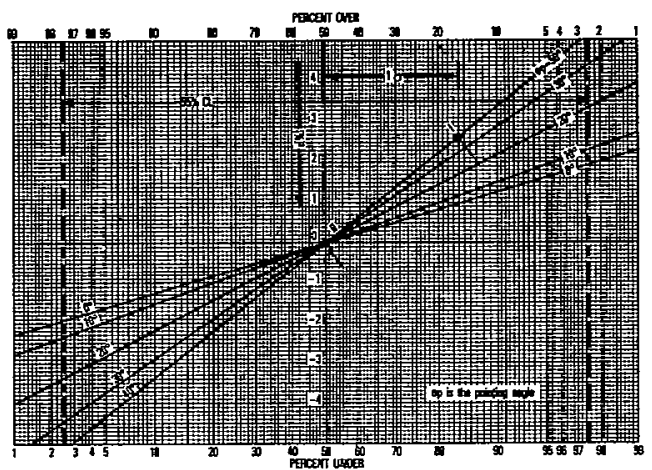

Figure 10 Plot of the Rin on probability paper

8. Conclusions and Recommendations Two error models for the $\mathrm{BS}^{3}$ have been developed. Either one or both may apply depending on whether the error sources are primarily deterministic or random or both. If the sources are deterministic then the accuracy and precision of the $\mathrm{BS}^{3}$ may be markedly improved through calibration and correcttive processes. If the sources are random then only statistical bounds may be defined for the system i.e., accuracy may be specified at thy desired confidence level.

It should be borne in mind that, although four error sources considered in this study are major sources, other sources are present and can only surve to further degenerate system performance. These other sources are believed to be minor so that their contributions are believed to be small. Since the specified exrox sources (SES) magnitudes given in Section 4 are estimates, laboratory/field measurement experiments designed to establish the ranges/statistics of the error sources are recommended.
The author would like to thank Mr. Richard Ribe of NOAA's Test and Evaluation Laboratory for evaluating the partials $p, a$, and $r$ (eq $5 a, 5 b$, $5 c)$ and for generating Table II.

\section{References}

[1] Angelari, Richard D. (National Ocean Survey, National Oceanic and Atmospheric Administration, Us. Department of Commerce, Rockville M. D.) 1978 "System Error Model of the Bathymetric Swath Survey System (BS 3 )" 77pp (unpublished manuscript) 